\title{
Expression analysis of the 35S CaMV promoter and its derivatives in transgenic hairy root cultures of cucumber (Cucumis sativus) generated by Agrobacterium rhizogenes infection
}

\author{
Mohammad Razi Anuar ${ }^{1}$, Ismanizan Ismail ${ }^{1,2 *}$ and Zamri Zainal ${ }^{1,2}$ \\ ${ }^{1}$ School of Biosciences and Biotechnology, Faculty of Science and Technology, Universiti Kebangsaan Malaysia, 43600 \\ Bangi, Selangor, Malaysia. \\ ${ }^{2}$ Center for Plant Biotechnology, Institute of System Biology, Universiti Kebangsaan Malaysia, 43600, Bangi, Selangor, \\ Malaysia.
}

Accepted 29 June, 2011

\begin{abstract}
The cauliflower mosaic virus (CaMV) 35 S promoter is the most commonly used viral-based promoter to drive transgene expression in plants. Although, many studies have demonstrated the constitutive nature of this promoter, some reports have suggested varied expression levels in different parts of the plant. Therefore, our aim was to study the activity of the CaMV 35S promoter in the hairy root system. The CaMV 35S promoter, the duplicate CaMV 35S promoter (designated CaMV 35ST) and the duplicate CaMV 35S promoter containing a 5'- untranslated leader sequence from the alfalfa mosaic virus RNA4 promoter (designated CaMV 35ST/AMV) were compared to evaluate their effects on the expression of the gus reporter gene in transgenic hairy roots, which was mediated using the Agrobacterium rhizogenes A4 transformation system. The integration of T-DNA containing a gus reporter gene in hairy root lines was confirmed at low copy numbers ranging from 1 to 4 copies using quantitative real-time PCR. Histochemical staining of cucumber hairy roots showed over-expression of the gus gene when driven with the CaMV 35S promoter. The expression of the gus gene when driven with the CaMV 35ST promoter showed a lower expression than that driven by the CaMV 35S promoter. However, the expression of the gus gene driven by the CaMV 35ST/AMV promoter was slightly higher than that driven by the CaMV 35ST promoter. In this study, the reduced activity of the CaMV 35ST promoter was observed for the first time. Further investigation is required to elucidate the factors that mediate the decline in promoter activity.
\end{abstract}

Key words: Cucumis sativus L, hairy root, Agrobacterium rhizogenes, promoter 35 S cauliflower mosaic virus (CaMV), $\beta$-glucuronidase (GUS), 5'UTR AMV.

\section{INTRODUCTION}

The development of genetic improvements in cucumbers has been used to produce desired traits and to study plant biological systems. The genetic transformation of

${ }^{*}$ Corresponding author. E-mail: maniz@ukm.my. Tel: +60 0389215954 . Fax: +60 0389252698.

Abbreviation: CaMV, Cauliflower mosaic virus; GUS, $\beta$ glucuronidase. cucumbers using Agrobacterium rhizogenes is not the optimal method to produce transgenic plants because the effects of this type of genetic transformation have not been well studied. In addition, it is difficult to regenerate transgenic plants from hairy root explants (Trulson et al., 1986; Yin et al., 2005). However, there are advantages to this transformation system when studying metabolic pathways of secondary metabolites and transgene expression in the hairy root system. The phenotype of the cucumber hairy root is plagiotropic and is characterized 
by numerous branches with rapid growth even in the absence of plant growth regulators (PGR) (Amselem and Tepfer, 1992). Transgenic hairy roots are genetically and biochemically stable, have a rapid growth rate and synthesize natural compounds at levels that are comparable to those in intact plants (Christey and Braun, 2005; Georgiev et al., 2007; Srivastava and Srivastava, 2007). These characteristics make the $A$. rhizogenes-mediated transformation system ideal for studying plant gene expression. Previous studies have reported on the use of the hairy root system (Peebles et al., 2007; Banarjee et al., 2002, 2008; Lee Flem-Bonhomme et al., 2004). Plant hairy roots display secondary metabolic 'manufacturing' capabilities that are suitable for the genetic engineering of metabolic pathways. Additionally, the hairy root system can produce or synthesize more than one type of secondary metabolite (Lee Flem-Bonhomme et al., 2004).

Genetically, engineered root cultures have been used as a model system to study various aspects of the metabolic and molecular regulation of several secondary metabolite pathways. For example, the over-expression of a cDNA encoding Panax ginseng farnesyl diphosphate synthase (FPS) in the hairy roots of Centella asiatica caused an increase in the contents of phytosterol and triterpene (Kim et al., 2010). Additionally, Lee et al. (2010) reported an increase in the rosmarinic acid level in the hairy roots of Nepeta cataria. The foreign genes that were introduced into the plant genomes were driven by a promoter that had been designed for expression in plants (Vengadesan et al., 2004).

A promoter is necessary to express genes newly introduced into plant DNA. In this study, three strategies were use to manipulate the expression of the reporter gene as follows: the $35 \mathrm{~S}$ CaMV promoter was used to drive the expression of the gus reporter gene in the plant; the duplicate 35S CaMV promoter was used to overexpress the reporter gene and the introduction of the 5'UTR of the alfalfa mosaic virus (AMV) sequence, an enhancer element, was used to enhance the translation process. The $35 \mathrm{~S}$ CaMV promoter is a well-known viral promoter that is constitutively active in several different species (Odell et al., 1985). Previous studies have indicated that the introduction of tandem repeats of the 35S CaMV promoter increases the expression levels by 100 fold compared with the nos promoter (Govindarajulu et al., 2008) in certain tissue types, but these expression levels were not studied in hairy root tissue. The tandem repeat of the 35S CaMV promoter acts as an enhancer element for the core promoter to increase promoter activities. Lam and Chua (1989) identified activation sequence 2 (as-2), which is homologous to the SV40 enhancer core A element and the Box II element of pea $r b c S$. The as-2 motif contributes to the expression in plant non-root parts and the as-1 motif contributes to the expression in the root and the protoplast (Lam et al., 1990). Interestingly, 35S CaMV elements can uniquely enhance or activate the activity of an adjacent tissue- and organ-specific gene promoter (Zheng et al., 2007).

The introduction of the AMV 5'UTR as a leader sequence was previously shown to enhance the translation efficiency of foreign genes (Gallie et al., 1987; Gallie and Walbot, 1992). A leader sequence functions to prevent the formation of secondary mRNA structures and provide a smoother translation process to increase the levels of the final gene product. Although, a previous study (Jobling and Gehrke, 1987) did not evaluate the effect of the leader sequence on the expression of a foreign gene in the hairy root system, our findings will establish a better understanding of the effects of the 5'UTR leader sequence in the expression of foreign genes in the hairy root. This approach should be useful for obtaining high expression levels of foreign genes in transgenic plants.

\section{MATERIALS AND METHODS}

The pCAMBIA 1301 binary vector was donated by Dr. Richard A. Jefferson at CAMBIA in Australia. The modified pCAMBIA 1301 vectors were constructed with the promoter modifications that are illustrated in Figure 1. The three promoters were transformed into Escherichia coli for propagation and confirmation purposes and were later transferred into the $A$. rhizogenes strain A4 before infecting the cotyledons of cucumbers. Figure 1 shows the preexisting promoters in PCAMBIA 1301 vector, which contains the 35S CaMV promoter in its T-DNA region and its modification to incorporate the 35ST CaMV and 35ST CaMV/AMV promoters. Cucumber seeds of the 'Simpang Pulai' variety were acquired from the Malaysian Agriculture, Research and Development Institute (MARDI), Malaysia.

\section{Explant preparation}

Cucumber transformation using each promoter was performed according to the transformation procedure that was previously described by Amselem and Tepfer (1992). Cucumber seeds were sterilized by shaking in $20 \%$ Clorox (Clorox, Kuala Lumpur, Malaysia) in sterile water for 20 min. Sterilized seeds were rinsed with sterile water, blotted dry and placed into Murashige and Skoog (MS) medium without PGRs. Seedlings were grown at $25^{\circ} \mathrm{C}$ for one week under a $16 \mathrm{~h}$ photoperiod until germination. Cotyledons of the sterile cucumber seedlings were excised and cultured in MS medium for 2 days under similar conditions.

\section{Plant transformation}

A. rhizogenes strain A4 cells harboring the binary vector pCAMBIA 1301 or one of its derivatives and carrying the gus reporter gene were grown to 0.5 to $0.6 \mathrm{OD}_{600}$ before co-cultivation. The cucumber cotyledons were transferred into a bacterial suspension for $20 \mathrm{~min}$. Then, the cotyledon explants were placed into co-culture medium containing MS with $200 \mu \mathrm{M}$ acetosyringone for 2 days at $25^{\circ} \mathrm{C}$ in the dark. Later, the cotyledons were transferred to MS medium containing $250 \mathrm{mg} / \mathrm{l}$ of cefotaxime (Duchefa, Haarlem, Netherland) to inhibit bacterial growth and they were cultured at $25^{\circ} \mathrm{C}$ with a 16 $\mathrm{h}$ photoperiod for two weeks. Two-centimeter-long hairy root initiates from the cotyledons were excised and placed onto MS medium containing $250 \mathrm{mg} / \mathrm{l}$ of cefotaxime to eliminate any residual bacterial under similar conditions. 


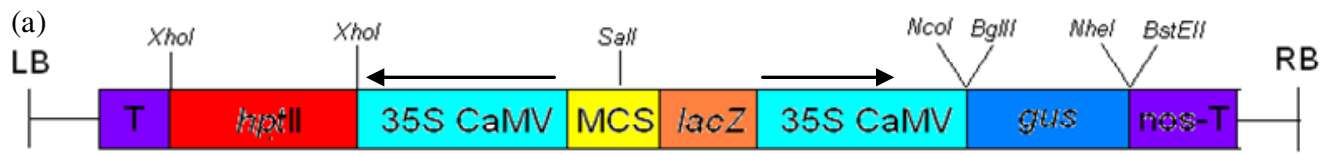

PCAMBIA 1301 T-DNA

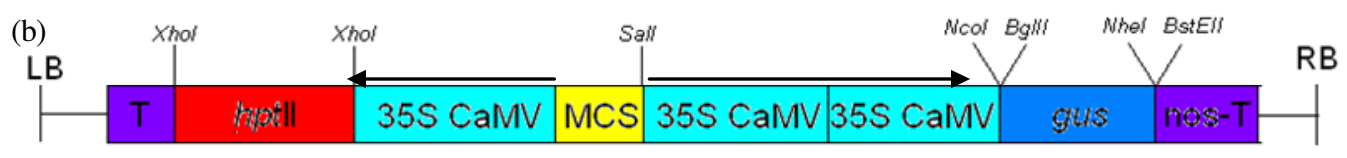

pCAMBIA 1301: promoter 35ST CaMV T-DNA

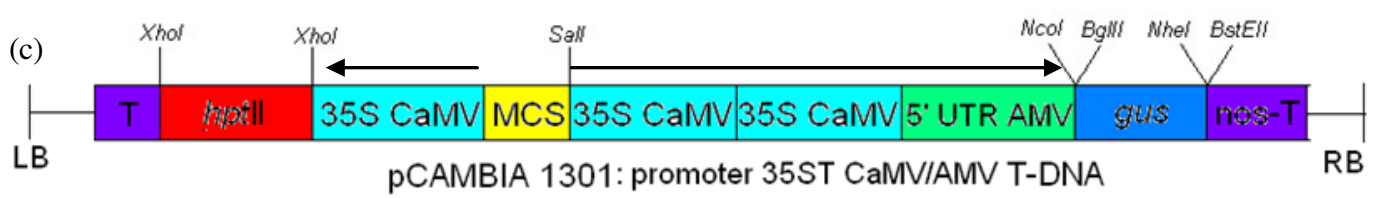

Figure 1. Linear map of PCAMBIA 1301 T-DNA region and modification of its 35S CaMV promoter. (a) T-DNA region for PCAMBIA 1301 with 35S CaMV promoter, (b) 35ST CaMV promoter replacing 35S CaMV promoter and (c) 35ST CaMV promoter with 5' UTR as leader sequence for gus gen. RB right border, $L B$ left border, hpt hygromicin phosphotransferase II, MCS multiple cloning sites, gus- $\beta$ Glucuronidase gen, nos- $T$ - nopaline terminator.

\section{Histochemical analysis of GUS expression}

The hairy root lines were tested using a histochemical GUS assay as previously described (Jefferson, 1987) to localize the distribution of GUS activity in transformed roots. One-centimeter-long hairy roots from each individual line were excised and transferred into histochemical assay buffer containing $0.1 \mathrm{M}$ phosphate buffer, 0.5 $\mathrm{mM}$ ferricyanide (Sigma, St. Louis, USA), $0.5 \mathrm{mM}$ ferrocyanide (Sigma, St. Louis, USA), 0.1\% Triton X-100 (Sigma, St. Louis, USA), $10.0 \mathrm{mM}$ EDTA (Sigma, St. Louis, USA), 20\% methanol (Sigma, St. Louis, USA) and $1.0 \mathrm{mM}$ 5-bromo-3-indolyl-glucuronide (X-gluc) (Duchefa, Haarlem, Netherlands). The samples were incubated for $16 \mathrm{~h}$ at $37^{\circ} \mathrm{C}$ in the dark. After staining, the sample solutions were removed and replaced with FAA solution [45\% absolute ethanol, (Hmbg, Hamburg, Germany), 5\% glacial acetic acid (Hmbg, Hamburg, Germany), 5\% formaldehyde (Hmbg, Hamburg, Germany)] for fixation. The samples were later examined under a light microscope.

\section{Fluorometric assay}

A fluorometric assay of gus gene expression was performed following the protocol that was provided with the FluorAce $\beta$ glucuronidase reporter assay kit (BioRad, Hercules, USA) to determine the activity of the gus gene by calculating the amount of methylumbelliferone (MU) present in the hairy root extract. The total protein content of the hairy root extract was determined using the Bradford assay (Bradford, 1976). Three replicates for each sample line were measured using a VersaFluor fluorometer (BioRad, Hercules, USA). For the negative control references, nontransgenic roots and hairy roots without the gus gene were subjected to analysis.

\section{PCR confirmation of transformants}

Ten putative positive lines for each promoter were subjected to
PCR using the gus and ro/B primers. The primer set for the gus gene was: F: 5'-CGCCGATGCAGATATTCGTA-3'; R: 5'-ATTAAT GCGTGGTCGTGCAC-3'. The primer set for the rolB gene was: F: 5'-TTAGGCTTCTTTCTTCAGGTTTACTGCAAGC-3'; R: 5'-ATGGA TCCCAAATTGCTATTCCTTCCACGA-3'.

Genomic DNA was isolated from each individual hairy root line using a previously described method (Doyle and Doyle, 1987). All of the PCR reactions were performed using a Mastercycler gradient (Eppendorf, Hamburg, Germany). Samples containing $200 \mathrm{ng}$ of genomic DNA were first heated at $94^{\circ} \mathrm{C}$ for $5 \mathrm{~min}$, followed by 30 cycles at $94^{\circ} \mathrm{C}$ for $1 \mathrm{~min}, 60^{\circ} \mathrm{C}$ (for the gus gene) or $57^{\circ} \mathrm{C}$ (for the ro/B gene) for $1 \mathrm{~min}$ and $72^{\circ} \mathrm{C}$ for $1 \mathrm{~min}$ followed by a 7 -min final extension at $72^{\circ} \mathrm{C}$. In total, $100 \mathrm{ng}$ of plasmid DNA was used as a positive control. The PCR reactions contained $200 \mu \mathrm{M}$ of each primer, $10 \mathrm{mM}$ dNTP mix, $15 \mathrm{mM} \mathrm{MgCl}_{2}, 50 \mathrm{mM} \mathrm{KCl}, 10 \mathrm{mM}$ Tris $\mathrm{HCl}(\mathrm{pH}$ 9.0), 0.1\% (v/v) Triton X-100, 2 U Taq DNA polymerase and $200 \mathrm{ng}$ of template DNA in $2 X$ reaction buffer. The amplified DNA was analyzed using $1.5 \%$ agarose gel electrophoresis.

\section{Quantitative real-time PCR}

Quantitative real-time PCR was performed according to the manufacturer's instructions using an IQ SYBR Green Supermix real-time PCR kit (BioRad, Hercules, USA). Quantitative real-time PCR was used to determine the copy number of the gus gene per genome in the transgenic cucumber hairy roots. The method of quantification used a standard curve for a series of copies of the gus gene. Plasmid pCAMBIA 1301 containing a single copy of gus gene per plasmid was prepared and the copy number of the gus gene was calculated per molecular weight. A primer set for realtime PCR was designed according to the manufacturer's instructions. The primer set for the gus gene was F: 5'-CAAC GGGGAAACTCAGCAAG-3'; R: 5'-AGCGTCGCAGAACATTACAT3'. Quantitative and relative real-time PCR were conducted using an IQ5 real-time PCR detection thermal cycler (BioRad, Hercules, USA). 

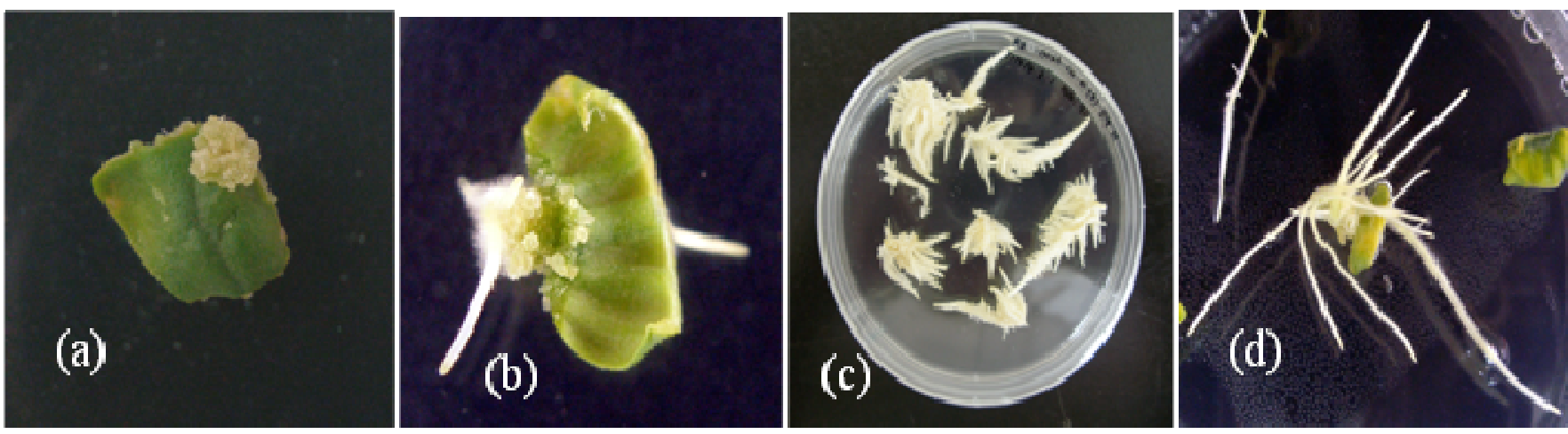

Figure 2. Picture of hairy root formation from wounded cucumber cotyledon after infection with $A$. rhizogenes. (a) Callus formation on wounding and infected site, (b) first hairy root initiation from callus, (c) elongation of hairy roots and (d) hairy root survived and growth without original cotyledon on MS medium without PGRs.

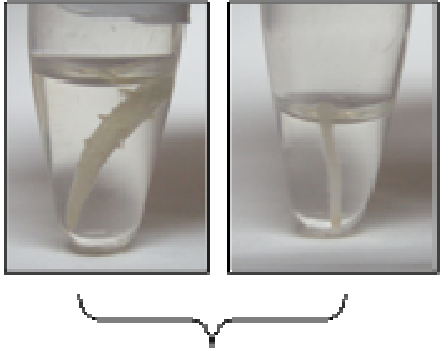

A

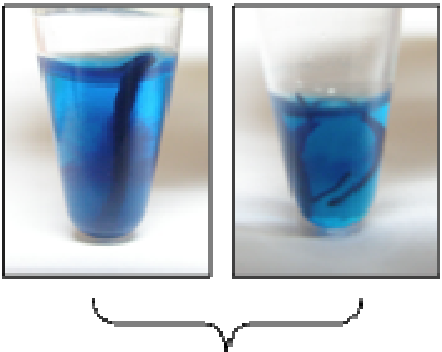

B

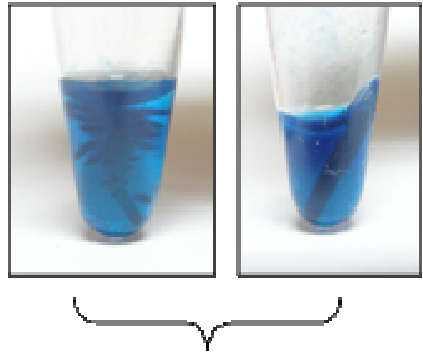

C

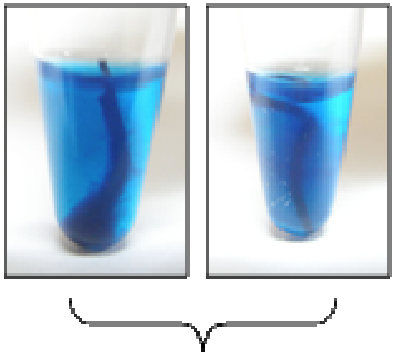

D

Figure 3. Early detection of gus gene expression via histochemical GUS assay after overnight incubation of hairy root lines. (A) Negative control, (B) hairy root lines with $35 \mathrm{~S}$ CaMV promoter, (C) hairy root lines with 35ST CaMV promoter and (D) hairy root lines with 35ST CaMV/AMV promoter.

\section{Relative real-time PCR}

Relative real-time PCR was used to determine the expression level of the GUS gene in each transgenic hairy root line, which was normalized to the level of an endogenous gene in cucumbers, the nadph-po (NADPH-protochlorophyllide oxidoreductase) gene. The cDNA from hairy roots that were transformed using $A$. rhizogenes A4 without any promoter was used to calibrate PCR products using a quantification method based on the Pfaffl method (Pfaffl, 2001). This method was chosen because the efficiency of the PCR reaction was in the acceptable range of 95 to $105 \%$ and the PCR process exponentially $\left(2^{n}\right)$ amplified cDNA templates. The primer set for the endogenous gene used was: $F$ : 5'-AAACG GCTCATCATTGTCG-3'; R: 5'-ATCGCTTGTGGAACTCTTGC-3'.

The total RNA was extracted from hairy root lines using an RNase plant mini kit (Qiagen, Germantown, USA) and was treated with RNase-free DNase-I to remove residual genomic DNA. The first-strand cDNA was synthesized using the RevertAid first strand cDNA synthesis kit (Fermentas, Burlington, Canada).

\section{RESULTS AND DISCUSSION}

The A4 strain of $A$. rhizogenes containing the binary vector was infectious to cucumbers. After 2 weeks, roots formed from explants and spread throughout the medium. These roots exhibited the same characteristics that have been previously reported (Mclnnes et al., 1991; Nader et al., 2006) and included abundant roots, auxiliary root growth and a plagiotropic phenotype. Figure 2 shows the formation of the hairy root phenotype from cucumber cotyledons after the transformation using $A$. rhizogenes.

Figure 3 shows histochemical GUS staining in transgenic hairy root lines. The presence of GUS activity and its localization were observed in all of the tissues of the root, especially in transgenic cucumber hairy root lines with the CaMV 35S and CaMV 35ST/AMV promoters. The observation of GUS activity was concentrated in the meristematic tissue and the vascular tissues of the root and the auxiliary roots. Figure 4 shows the specific localization of GUS activity in different tissue types. The transgenic cucumber hairy roots lines with the CaMV 35S promoter or the CaMV 35ST promoter showed localized GUS activity only in the vascular bundles.

Benfey and Chua (1990) reported that 2 domains in the 35S promoter confer gene expression in different tissues; domain A confers gene expression in meristematic tissue 

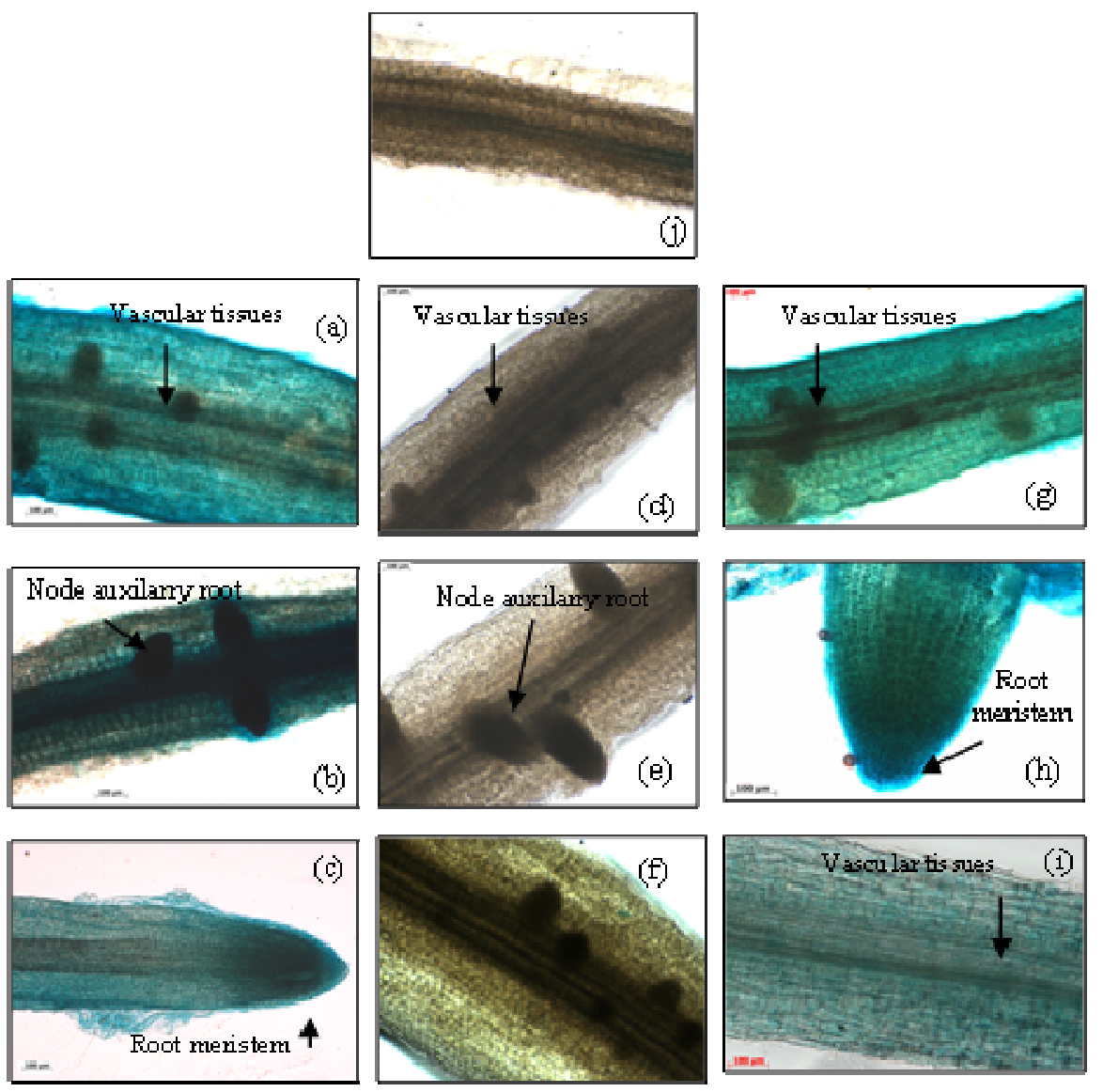

Figure 4. Picture from above, (a), (b) and (c); GUS distribution in hairy root lines transformed with 35S CaMV promoter, (d), (e) and (f); hairy root lines with 35ST promoter, (g), (h) and (i); hairy root lines with 35ST/AMV promoter. (j); negative control of hairy root transform with $A$. rhizogenes wild type.

and domain B confers gene expression in vascular bundles. Their report suggests that using the full-length 35S CaMV promoter may confer gene expression in these tissue types, while using the duplicate repeat of the 35S CaMV promoter was expected to increase the gene expression in these tissues. In this study, we demonstrated that using the tandem repeat promoter sequence reduced the promoter activity and conferred gene expression only in the vascular bundles and not in the root meristematic tissue.

A fluorometric GUS assay was performed to determine the expression level in transgenic hairy root lines that were previously analyzed using a histochemical GUS assay. Cucumber roots and cucumber hairy roots that were transformed using $A$. rhizogenes without a binary vector were used as negative controls. The GUS activity for each transgenic hairy root line with each promoter is shown in Figure 5. Based on the fluorometric results, we showed that the highest GUS expression in the hairy root line was in the plants transformed using the 35S CaMV promoter $(3288 \mathrm{pmol} / \mu \mathrm{g} / \mathrm{min})$, whereas the hairy root line that was transformed using the 35ST CaMV promoter showed the lowest GUS expression (353 pmol/ $\mu \mathrm{g} / \mathrm{min}$ ). Very low endogenous GUS activity was detected in the control samples. These results show that cucumber hairy root tissue exhibits very low or negligible endogenous GUS expression and is suitable for the gus reporter gene studies. Jefferson and Wilson (1991) have suggested that higher plants show low or no GUS activity all endogenously.

PCR confirmation of the gus and ro/B gene expression profiles (Table 2) revealed the presence of both genes in all of the putative transgenic hairy root lines. This result shows that the T-DNA region of the binary vector and $\mathrm{pRi}$ was successfully transferred into plant cells. The integration of the gus gene was confirmed using quanti-tative RT-PCR.

Quantification of the copy number of the gus gene using absolute quantification in real-time PCR revealed a low copy number of the GUS gene per genome. The results of this quantification are shown in Table 1. Figure 6 shows the copy number of the gus gene in each transgenic hairy root line. The determination of the gene copy number was required to gauge the level of gene 
GUS activity (pmol/ug/min)

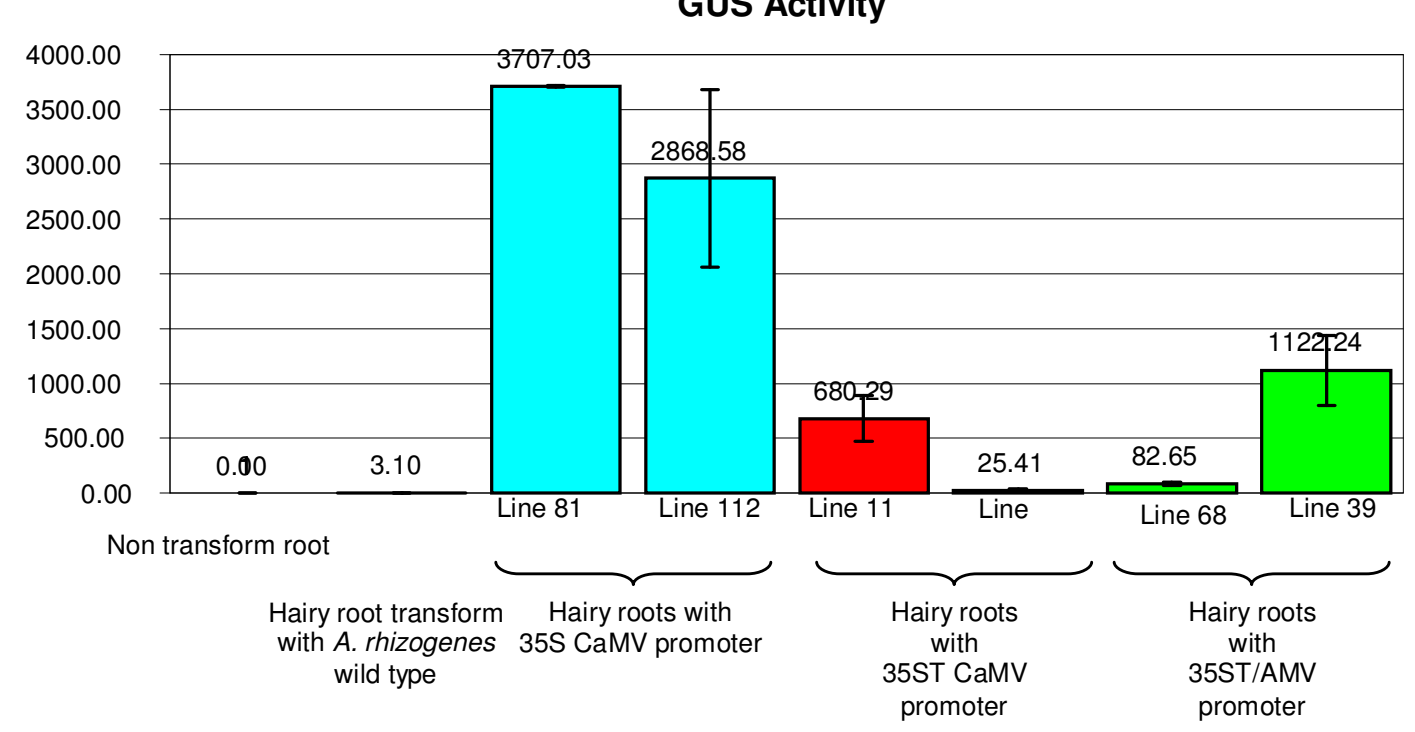

Figure 5. A fluorometric GUS assay shows the pattern of expression for each tested hairy root line with different promoters

Table 1. Quantitative real-time PCR result for gus gene quantification per genome.

\begin{tabular}{lc}
\hline Cucumber hairy root line & Copy/genome \\
\hline 81 with CaMV 35S promoter & 4 \\
112 with CaMV 35S promoter & 1 \\
14 with CaMV 35ST promoter & 1 \\
11 with CaMV 35ST promoter & 1 \\
68 with CaMV 35ST/AMV promoter & 1 \\
39 with CaMV 35S/AMV promoter & 1 \\
\hline
\end{tabular}

Table 2. PCR confirmation for putative positive lines of hairy root harbouring particular promoters.

\begin{tabular}{lccccc}
\hline \multirow{2}{*}{ Promoter } & $\begin{array}{c}\text { Number of positive line from } \\
\text { Histochemical GUS/ actual line tested }\end{array}$ & \multicolumn{2}{c}{$\begin{array}{c}\text { Number of line } \\
\text { performing PCR }\end{array}$} & \multicolumn{2}{c}{$\begin{array}{c}\text { Number of line successfully } \\
\text { confirmed through PCR }\end{array}$} \\
\cline { 3 - 6 } & & GUS & RolB & GUS & RolB \\
\hline 35S CaMV & $18 / 300$ & 11 & 11 & 10 & 11 \\
35ST CaMV & $20 / 300$ & 10 & 10 & 10 & 10 \\
35ST CaMV/ AMV & $75 / 300$ & 9 & 10 & 9 & 9 \\
\hline
\end{tabular}

expression. Kohli et al. (1999) reported that a high rate of foreign gene insertion may contribute to the instability or a reduced level of gene expression. Fortunately, Agrobacterium-based transformation induces a low rate of foreign gene insertion as suggested by Gelvin (2003). Kohli et al. (1999), Dong et al. (2001) and Khristaleve and Kik, (2001) reported that the integration of foreign genes is random and that foreign genes tend to integrate into distal chromosomal regions of the genome.

Similar to our observations in the fluorometric assay, relative real-time PCR also showed a similar pattern of gene expression for each of the transgenic hairy root lines. This result shows that the transcription and posttranslation of the gus gene are consistent with our findings. The results of the relative real-time PCR are shown in Figure 6. The highest recorded difference in the gene expression was seen in transgenic plants with the 35S CaMV promoter (14-fold difference compared with that of wild-type plants). The lowest recorded difference in the gene expression was seen in transgenic plants with 


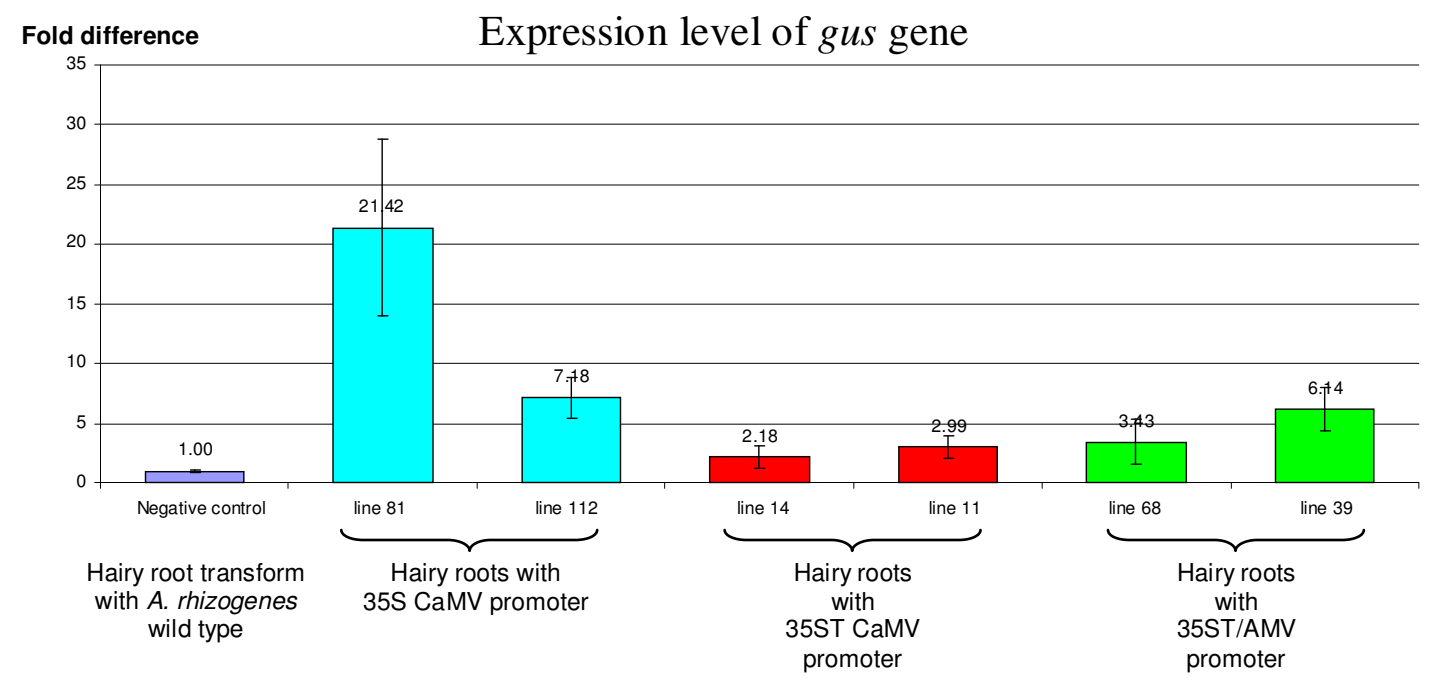

Figure 6. Relative real-time PCR results for each corresponding promoter constructed in cucumber hairy root lines.

the 35ST CaMV promoter (2.6-fold difference compared with that of wild-type plants).

Based on the previous studies of 35S CaMV promoter, we expected a high level of expression of the gus gene (Kay et al., 1987; Omirulleh et al., 1993; Govindarajulu et al., 2008). Previous studies have shown that the duplicate $35 \mathrm{~S}$ CaMV promoter induces a greater increase in the gene expression when compared with the 35S CaMV promoter alone. Interestingly, we found a reduced expression of the reporter gene when using the duplicate 35S CaMV promoter. This report is the first to identify the reduced activity of the duplicate $35 \mathrm{~S}$ CaMV promoter in the hairy root system.

Histochemical staining of putative positive cucumber hairy roots showed the over-expression of the gus gene when the expression was driven by the $35 \mathrm{~S}$ CaMV promoter. These findings were confirmed using a fluorometric GUS assay and relative real-time PCR. The expression of the gus gene driven by the CaMV 35ST promoter was lower than that driven by the CAMV 35S promoter. GUS expression driven by the CaMV 35ST promoter displayed a decrease of 9.3 fold using the fluorometric GUS assay (Figure 5) and a decrease of 5.5 fold using relative real-time PCR (Figure 6). The expression of the gus gene driven by the CaMV 35ST/AMV promoter was slightly increased compared with that driven by the CaMV 35ST promoter; the expression level was found to be increased by 1.7 fold using the fluorometric GUS assay (Figure 5) and by 1.9 fold using relative real-time PCR (Figure 6). Differences in the fold change between the fluorometric GUS assay and relative real-time PCR may be attributed to differences in the stages of gene expression at the time of the analysis. The fluorometric analysis detected the expression at the translation level, whereas the relative real-time PCR compared the expression at the transcription level.
Figures 5 and 6 show that even though the hairy root lines harbored similar promoters, individual transgenic hairy root lines produced varied expression levels that may be regulated by internal and external factors. Therefore, the mean expression activity was used to represent lines containing the promoter of interest. The variation in foreign gene expression in transgenic hairy roots may have contributed to differences in the localization of gene integration, the silencing effects during transcription or post-translation or the effects of endogenous protein factors (Jorgensen et al., 1996; Wakimoto, 1998; Wagner and Garcia-Blanco, 2001; Schubert et al., 2004).

The introduction of the tandem 35S CaMV promoter resulted in reduced expression levels of the gus gene and potentially contributed to the absence of motifs or sequences that were necessary for elevated gene expression in cucumber hairy roots. The 35ST promoter has a double -343 to -46 sequences, which is the optimal sequence that is required for the elevated expression in planta (Odell et al., 1985). However, the full-length 35S CaMV promoter contains a downstream sequence from 343 that may contain unknown elements that may increase the expression of genes, especially in the cucumber hairy root system. Further studies are required to test the hypothesis that unknown elements downstream of the -343 sequence of the $35 \mathrm{~S}$ CaMV promoter may contribute to the regulation of gene expression.

The introduction of the 5'UTR of AMV increased gus gene activity. The results from the fluorometric assay and relative RT-PCR indicate that the AMV sequence enhanced gene expression at the transcriptional and translational levels. The AMV sequence, similar to the TMV $\Omega$ leader sequence, exhibits a translational enhancer capability such that this sequence improves the stability and the translation efficiency of mRNA (Kozak, 
1987; Gallie, 1991; Gallie and Walbot, 1992). The results show that the 5'UTR sequence can be used to enhance foreign gene expression in the hairy root system, especially in cucumbers.

\section{Conclusion}

In this study, the expression of the gus gene was successfully elevated and showed increased expression in cucumber hairy roots. Although, our strategy to modify the CaMV 35S promoter was not successful, we showed that the 35S CaMV and AMV enhancer elements strongly affected the expression of the gus gene in the cucumber hairy root. This approach is beneficial for understanding the root system and is an excellent model for $A$. rhizogenes-mediated transformation.

\section{ACKNOWLEDGEMENT}

This research was funded by IRPA Grant 02-01-02SF0143 from the Ministry of Science, Technology and Innovation (MOSTI) Malaysia awarded to Ismanizan Ismail. In addition, we thank Tee Choon Yang for his critical reading of this manuscript.

\section{REFERENCES}

Amselem J, Tepfer M (1992) Molecular basis for novel root phenotypes induced by Agrobacterium rhizogenes A4 on cucumber. Plant Mol. Biol. 19: 421-432.

Banarjee S, Shang TQ, Wilson AM, Moore AL, Strand SE, Gordon MP, Doty SL (2002). Expression of functional mammalian p450 2E1 in hairy root cultures. Biotechnol. Bioeng. 77(4): 462-466.

Banerjee S, Madhusudanan KP, Chattopadhyay SK, Rahman LU, Khanuja SPS (2008). Expression of tropane alkaloids in the hairy root culture of Atropa acuminata substantiated by DART mass spectrometric technique. Biomed. Chromatography, 22(8): 830-834.

Benfey PN, Chua NH (1990). The Cauliflower Mosaic Virus 35S promoter: Combinatorial regulation of transcription in plants. Science, 250: 959-966.

Bradford MM (1976). A rapid sensitive method for the quantitation of microgram quantities of protein utilizing the principle of protein-dye binding. Anal. Biochem. 72: 248-254.

Christey MC, Braun RH (2005). Production of hairy root cultures and transgenic plants by Agrobaterium rhizogenes-mediated transformation. Methods Mol. Biol. 286: 1283-1288.

Dong JJ, Kharb P, Teng WM, Hall TC (2001). Characterization of rice transformed via an Agrobacterium-mediated inflorescence approach. Mol. Breed. 7: 187-194.

Doyle JJ, Doyle JL (1987). A rapid DNA isolation procedure for small quantities of fresh leaf tissues. Phytochem. Bull. 19: 11-15.

Gallie DR (1991). The cap and poly(A) tail function synergistically to regulate mRNA translational efficiency. Genes Dev. 5: 2108-2116.

Gallie DR, Sleat DE, Watts JW, Turner PC, Wilson TMA (1987). The 5'leader sequence of tobacco mosaic virus RNA enhances the expression of foreign gene transcripts in vitro and in vivo. Nucleic Acids Res. 15: 3257-3273.

Gallie DR, Walbot V (1992). Identification of the motifs within the tobacco mosaic virus 50-leader responsible for enhancing translation. Nucleic Acids Res. 20: 4631-4638.

Gelvin SB (2003). Agrobacterium-Mediated Plant Transformation: the Biology behind the Gene-Jockeying Tool. Microbiol. Mol. Biol. Rev.
$6(1)$

Georgiev MI, Pavlov Al, Bley T (2007). Hairy root type plant in vitro systems as sources of bioactive substances. Appl. Microbiol. Biotechnol. 74: 1175-1185.

Govindarajulu M, Elmore JM, Fester T, Taylor CG (2008). Evaluation of Constitutive Viral Promoters in Transgenic Soybean Roots and Nodules. Molecular Plant-Microbe Interactions, 21(8): 1027-1035.

Jefferson AR (1987). Assaying chimeric genes in plants: the GUS gene fusion system. Plant Mol. Biol. Rep., 5: 387-405.

Jefferson RA, Wilson KJ (1991). The GUS gene fusion system. Plant Mol. Biol. Man, 14: 1-33.

Jobling SA Gehrke L (1987). Enhanced translation of chimeric messenger RNAs containing a plant viral untranslated leader sequence. Nature, 325: 622-624.

Jorgensen RA, Cluster PD, English J, Que Q, Napoli CA (1996). Chalcone synthase cosuppression phenotypes in petunia flowers: comparison of sense vs. Antisense constructs and sigle-copy vs. complex T-DNA sequences. Plant Mol. Biol. 31(5): 957-973

Kay R, Chan A, Daly M, McPherson J (1987). Duplication of CaMV 35 S promoter sequences creates a strong enhancer for plant genes. Science. 236: 1299-1302.

Khristaleve LI, Kik C (2001). Localization of single-copy T-DNA insertion in transgenic shallots (Allium cepa) by using ultra-sensitive FISH with tyramide signal amplification. Plant J. 25: 699-707.

Kim O, Kim S, Ohyama K, Muranaka T, Choi Y, Lee H, Kim M, Hwang $\mathrm{B}$ (2010). Upregulation of phytosterol and triterpene biosynthesis in Centella asiatica hairy roots overexpressed ginseng farnesyl diphosphate synthase. Plant Cell Rep. 29: 403-411.

Kohli A, Griffiths S, Palacios N, Twyman RM, Vain P, Laurie DA, Christou P (1999). Molecular characterisation of transforming plasmid rearrangements in transgenic rice reveals a recombination hotspot in the CaMV 35S promoter and confirms the predominance of microhomology-mediated recombination. Plant J. 17(6): 591-601.

Kozak M (1987). An analysis of 5'-noncoding sequences from 699 vertebrate messenger RNAs. Nucl Acids Res. 15: 8125-8132.

Lam E. Chua NH (1989). ASF-2: a factor that binds to the cauliflower mosaic virus $35 \mathrm{~S}$ promoter and a conserved GATA motif in Cab promoters. Plant Cell, 1(12): 1147-1156.

Lam E, Benfey P, Chua NH (1990). Characterization of as-1: a factor binding site on the $35 \mathrm{~S}$ promoter of cauliflowe mosaic virus. UCLA Symposia Mol. Cell. Biol. 129: 71-79.

Lee Flem-Bonhomme V, Laurain-Mattar D, Fliniaux MA (2004). Hairy root induction of Papaver somniferum var. album, a difficult-totransform plant, by A. rhizogenes LBA 9402. Planta, 218: 890-893.

Lee SY, Lee CY, Eom SH, Kim YK, Park NI, Park SU (2010). Rosmarinic acid production from transformed root cultures of Nepeta cataria L. Sci. Res. Essays, 5(10): 1122-1126.

McInnes E, Morgan AJ, Mulligan BJ, Davey MR (1991). Roots induced on cucumber cotyledons by the agropine Ri plasmid TR-DNA exhibit the transformed phenotype. Plant Cell Rep. 9: 647-650.

Nader BL, Taketa AT, Pereda-Miranda R, Villarreal ML (2006). Production of triterpenoids in liquid-cultivated hairy roots of Galphimia glauca. Planta Med. 72(9): 842-844.

Odell JT, Nagy F, Chua NH (1985). Identification of DNA sequences required for activity of the cauliflower mosaic virus $35 \mathrm{~S}$ promoter. Nature, 313, 810-812.

Omirulleh S, Abraham M, Golovkin M, Stefanov I, Karabaev MK, Mustardy L, Morocz S, Dudits D (1993). Activity of chimeric promoter with the doubled CaMV $35 \mathrm{~S}$ enhancer element in protoplast-derived cells and transgenic plants in maize. Plant Mol. Biol. 21(3): 415-428.

Peebles CAM, Gibson SI, Shanks JV and San KY (2007). Long-Term Maintenance of a Transgenic Catharanthus roseus Hairy Root Line. Biotechnol. Progress. 23(6): 1517-1518

Pfaffl MW (2001). A new mathematical model for relative quantification in real-time RT-PCR. Nucleic Acids Res. 29(9): 2002-2007.

Schubert D, Lechtenberg B, Forsbach A, Gils M, Bahadur S, Schmidt R (2004). Silencing in Arabidopsis T-DNA transformants: The predominant role of a gene-specific RNA sensing mechanism versus position effects. Plant Cell. 16: 2561-2572.

Srivastava S, Srivastava AK (2007). Hairy root culture for massproduction of high-value secondary metabolites. Crit. Rev. Biotechnol. 27: 29-43. 
Trulson AJ, Simpson RB, Shahin EA (1986). Transformation of cucumber (Cucumis sativus L.) with Agrobacterium rhizogenes. Theor. Appl. Gen., 73: 11-15.

Vengadesan G, Anand RP, Selvaraj N, Perl-Treves R, Ganapathi A (2004). Transfer and expression of nptll and bar genes in cucumber (Cucumis sativus L.). In vitro Cell. Dev. Biol. 41(6): 789-793.

Wagner EJ, Garcia-Blanco MA (2001). Polypyrimidine tract binding protein antagonizes exon definition. Mol. Cell. Biol. 21(10): 32813288.

Wakimoto BT (1998). Beyond the nucleosome: Epigenetics aspects of position-effect variegation in Drosophila. Cell, 93: 321-324.

Yin Z, Bartoszewski G, Szwacka M, Malepszy S (2005). Cucumber transformation methods-the reviews. Biotechnology, 1(68): 95-113
Zheng X, Deng W, Luo K, Duan H, Chen Y, McAvoy R, Song S, Pei Y, $\mathrm{Li} Y$ (2007). The cauliflower mosaic virus (CaMV) 35S promoter sequence alters the level and patterns of activity of adjacent tissueand organ-specific gene promoters. Plant Cell Rep., 26: 1195-1203. 